Conclusions: This study highlights the impact RA has on working life amongst both those with the condition and those providing support. It is hoped these metrics will allow the conversation to open and develop with employers and government on how adapting the workplace can increase productivity. Further research should also be undertaken on the economic impact both to the individuals, their carers and society.

Acknowledgements: The BRASS study was supported by unrestricted research grants from Eli Lilly and Pfizer. The study was approved by the University of Chester Ethics Committee.

Disclosure of Interest: None declared

DOI: 10.1136/annrheumdis-2017-eular.5948

\section{OP0318-PARE CONSULTING SERVICES TO EMPLOYERS OF PEOPLE WITH RMDS}

F.J. Carreira Roca ${ }^{1,2}$, A.I. Vázquez Lojo ${ }^{3}$, V. Romero Pazos ${ }^{4,5}$ on behalf of LIRE-LRG. ${ }^{1}$ Accesibility Dept. and Volunteer, Liga Reumatolóxica Galega (LRG), A Coruña; ${ }^{2}$ Accesibility Consultant and Volunteer, Liga Reumatológica Española (LIRE), Madrid; ${ }^{3}$ Board of Directors and Occupational Therapy Dept., Liga Reumatolóxica Galega; ${ }^{4}$ International Dept., Liga Reumatolóxica Galega (LRG), A Coruña; ${ }^{5}$ Board of Directors, Liga Reumatológica Española (LIRE), Madrid, Spain

Background: The Eurobarometer ${ }^{1}$ states that RMDs affect $22 \%$ of the general population which means that 10 million people are affected in Spain and more than 600.000 people in the autonomous region of Galicia where our organization, LRG, works.

Knowing that $50 \%$ of people with RMDs are currently unemployed because of their condition ${ }^{2}$, only part of them have the legal work incapacity recognized and receive a pension. This means that RMDs not only affect the health but also the socio-economic status. Most of this people could stay at work and are willing to do so if some arrangements are made like cutting down hours, work place adaptations, flexible schedule, etc. ${ }^{3}$

Most employers are lacking information on how these conditions affect the employee's hability, desire and need to work and how to make the necessary adaptations for not loosing this person from the work force.

Objectives: Making the employer more aware of the positive aspects of keeping all the employees including people with RMDs not just at the work place but at full capacity, avoiding both absenteeism and presenteeism.

Helping the employer knowing about the needs of people with RMDs and the necessary adaptations for each of them.

Keep people with RMDs at work at the best possible conditions both for the employee and the employer, as efficiently and healthy as possible.

Methods: The employee with RMD has 1 session with the psychologist and 1 session with the occupational therapist (OT) to identify the difficulties in continuing to be active into their work place.

LRG HP give personalized advise to the employer about the RMDs, the needs of the employees and how to facilitate them to stay at work and minimize the work incapacity due to their chronic condition. This kind of advise is given by the OT who has treaten the employees and knows all about their needs, goes to their work place to see what are the actual conditions, identifies manageable obstacles and talks to the employers during a pre-scheduled meeting. After, an architect specialized in accesibility offers specific architectural and ergonomic solutions for adapting the work place.

Also peer support is provided by LRG in group meetings and activities of the organization.

Results: The results of this project are shown in the increase of the employees' self-confidence in their capacity to stay at work and also they are more aware of their solvable and non solvable limitations.

During 2016 we advised 14 associates of LRG, 5 of those 14 employees (1/3) stayed at work or returned to work after the consultancy.

Conclusions: This kind of actions are neccesary to make the work places more adaptable and increasing the number of people with RMDs to start a work live, to stay or go back to work.

Peer support within a patient's association is key to create a secure environment for the employees who find relief in seeing other people with RMDs having a sustainable work life.

References:

[1] European Commission. Health in the European Union. Special Eurobarometer 272e. 2007.

[2] Anna Klak, Filip Raciborski and Piotr Samel-Kowalik. Social implications of rheumatic diseases. Reumatologia 2016; 54(2): 73-78.

[3] Bevan, S., Passmore, E., Mahdon, M. (2007). Fit for Work? Musculoskeletal Disorders and Labour Market Participation. UK: The Work Foundation.

Acknowledgements: Employers: SERGAS, Correos, Inditex Group, Estrella Galicia.

Disclosure of Interest: None declared

DOI: 10.1136/annrheumdis-2017-eular.6342

\section{FRIDAY, 16 JUNE 2017 \\ Biomarkers in cardiovascular rheumatology - state-of-the-art 2017}

\section{OP0319 HIGH SENSITIVITY CARDIAC TROPONIN T IS A BIOMARKER FOR ATHEROSCLEROSIS IN SYSTEMIC LUPUS ERYTHEMATOUS PATIENTS: A CROSS-SECTIONAL CONTROLLED STUDY}

G. Divard ${ }^{1}$, R. Abbas ${ }^{1}$, C. Chenevier-Gobeaux ${ }^{2}$, B. Escoubet ${ }^{1}$, M.-P. Chauveheid ${ }^{1}$, A. Dossier ${ }^{1}$, M. Dehoux ${ }^{1}$, T. Papo ${ }^{1}$, K. Sacre ${ }^{1} .{ }^{1}$ Paris-7 University, APHP, Bichat Hospital; ${ }^{2}$ Paris-5 University, APHP, Cochin Hospital, Paris, France

Background: Cardiovascular disease (CVD) is the main cause of death in systemic lupus erythematous (SLE) patients. Framingham score underestimates the risk for CVD in this population.

Objectives: Our study aimed to determine whether serum High Sensitivity Cardiac Troponin T (HS-cTnT) helps to identify SLE patients at risk for CVD.

Methods: Presence of carotid plaques was prospectively assessed by ultrasound in 63 consecutive SLE patients asymptomatic for CVD and 18 controls. Serum HScTnT concentration was measured using the electrochemiluminescence method. Factors associated with carotid plaques were identified and multivariate analysis was performed

Results: Framingham score was low in both SLE patients $(2.1 \pm 3.8 \%)$ and controls $(2.1 \pm 2.9 \%)$. Nevertheless, $23(36.5 \%)$ SLE patients, but only $2(11.1 \%)$ controls $(\mathrm{p}=0.039)$, had carotid plaque detected by vascular ultrasound. In the multivariate analysis, only age $(p=0.006)$ and SLE status $(p=0.017)$ were independently associated with carotid plaques. Serum HS-cTnT concentration was detectable (i.e. $>3 \mathrm{ng} / \mathrm{L})$ in $37(58.7 \%)$ SLE patients and $6(33.3 \%)$ controls $(p=0.057)$. Interestingly, $87 \%$ of SLE patients with carotid plaques, but only $42.5 \%$ in SLE patients without plaques $(p<0.001)$, had a detectable HS-cTnT. Conversely, $54.5 \%$ SLE patients with a detectable HS-cTnT, but only $11.5 \%$ with an undetectable HS-cTnT $(p<0.001)$, had a carotid plaque. In the multivariate analysis, only BMI $(p=0.006)$ and HS-cTnT $(p=0.033)$ were statistically associated with carotid plaques in SLE patients. Overall, the risk of having a carotid plaque was increased by 8 (OR [95\% Cl]: 8.03 [1.41-74.73]) in SLE patients in whom HS-cTnT was detectable in serum.

Conclusions: Detectable HS-cTnT concentration is independently associated with subclinical atherosclerosis in asymptomatic SLE patients at apparent low risk for CVD according to traditional risk factors. These results raise the possibility that this easily obtained biomarker is useful for more rigorous risk stratification and primary prevention of CVD in SLE patients.

Disclosure of Interest: None declared

DOI: 10.1136/annrheumdis-2017-eular.1015

\section{FRIDAY, 16 JUNE 2017}

\section{What is behind vasculitis?}

\section{OP0320 DETERMINANTS OF RITUXIMAB PHARMACOKINETICS AND CLINICAL OUTCOMES IN PATIENTS WITH ANCA-ASSOCIATED VASCULITIS}

D. Cornec $^{1,2}$, B. Kabat ${ }^{1}$, J. Mills ${ }^{1}$, M. Cheu $^{3}$, A. Hummel $^{1}$, D. Schroeder ${ }^{1}$, M. Cascino ${ }^{3}$, P. Brunetta ${ }^{3}$, D. Murray ${ }^{1}$, M. Snyder ${ }^{1}$, F. Fervenza ${ }^{1}$, G. Hoffman ${ }^{4}$ C. Kallenberg ${ }^{5}$, C. Langford ${ }^{4}$, P. Merkel ${ }^{6}$, P. Monach ${ }^{7}$, P. Seo ${ }^{8}$, R. Spiera ${ }^{9}$, W.St. Clair ${ }^{10}$, S. Ytterberg ${ }^{1}$, J. Stone ${ }^{11}$, D. Barnidge ${ }^{1}$, U. Specks ${ }^{1} .{ }^{1}$ Mayo Clinic, Rochester MN, United States; ${ }^{2}$ Rhumatologie, CHRU Brest, Brest, France; ${ }^{3}$ Genentech Inc., San Francisco, CA; ${ }^{4}$ Cleveland Clinic, Cleveland $\mathrm{OH}$, United States; ${ }^{5}$ Univeristy, Groningen, Netherlands; ${ }^{6}$ University of Pennsylvania, Philadelphia PA; ${ }^{7}$ Boston University, Boston MA; 8 Johns Hopkins, Baltimore MD ${ }^{9}$ Hospital for Special Surgery, New York; ${ }^{10}$ Duke University, Durham NC;

${ }^{11}$ Massachusetts General Hospital, Boston MA, United States

Background: Response to rituximab (RTX) is variable in patients with ANCAassociated vasculitis (AAV), and predictors of treatment efficacy/relapse risk would be useful. Previous studies have shown that RTX pharmacokinetics (PK) is associated with treatment efficacy in patients with lymphoma.

Objectives: To study the determinants of RTX PK in patients treated for AAV and its association with clinical outcomes.

Methods: This study included 88 patients from the RTX in ANCA-Associated Vasculitis (RAVE) trial who received the full dose of RTX (4 weekly $375 \mathrm{mg} / \mathrm{m}^{2}$ infusions) and had available serum samples. RTX was quantified using two different assays: a traditional ELISA and a recently developed mass spectrometrybased assay (referred to as miRAMM). We analyzed week (W)2, W4, W8, W16 and W24 serum levels and the trapezoidal area under the curve (AUC) integrating baseline, W2, W4, and W8 levels. We explored potential determinants of RTX PK using univariate and multivariate analysis, and analyzed the association of RTX PK with clinical outcomes: achievement of complete remission at 6 months (defined by a BVAS/WG score of 0 with no prednisone), time to relapse in patients who achieved complete remission, and B-cell depletion duration. 
Results: RTX quantifications using ELISA and miRAMM were highly correlated, but miRAMM measured consistently higher serum levels, suggesting its ability to detect total serum RTX (including free and complexed rituximab). RTX PK was highly variable between patients, with W2 levels ranging between 43 and 259 $\mu \mathrm{g} / \mathrm{mL}$ and AUC ranging between 2,668 and $17,513 \mu \mathrm{g} / \mathrm{mL}$ by miRAMM. W2 RTX levels and AUC were significantly lower in males and in newly-diagnosed patients, and were negatively correlated with body surface area, baseline B-cell count, and BVAS/WG. In multivariate analyses, the main determinants of RTX PK were sex and new diagnosis. Patients with a new diagnosis had higher baseline B-cell counts and BVAS/WG. Patients reaching complete remission at month 6 had similar mean RTX levels compared to patients who did not reach complete remission (W2 level by miRAMM: $136 \pm 44$ vs $139 \pm 46, p=0.76$; AUC: $8420 \pm 2875$ vs $8558 \pm 3452, p=0.85)$. Patients with higher RTX levels generally experienced longer B-cell depletion durations, but RTX levels at the different time-points and AUC were not associated with time to any relapse or time to severe relapse. Similar results were observed when using rituximab quantification by miRAMM and by ELISA.

Conclusions: Despite a dosing protocol adjusted for body surface area, rituximab PK is highly variable between patients, its main determinants being sex and newly diagnosed disease. We did not observe an association between rituximab PK and clinical outcomes. Serum rituximab level monitoring does not seem clinically useful in this context.

Disclosure of Interest: None declared

DOI: 10.1136/annrheumdis-2017-eular.3527

\section{OP0321 RISK OF CORONARY ARTERY DISEASE AND ISCHEMIC STROKE IN PATIENTS WITH ANCA-ASSOCIATED VASCULITIS.} A FRENCH POPULATION-BASED STUDY

M. Mourguet ${ }^{1}$, D. Chauveau ${ }^{2}$, A. Huart ${ }^{3}$, D. Ribes ${ }^{4}$, S. Faguer ${ }^{5}$, L. Sailler ${ }^{1}$, G. Pugnet ${ }^{1} .{ }^{1}$ Service de Médecine Interne, $\mathrm{CHU}$ Toulouse Purpan;

${ }^{2}$ Département de Néphrologie et transplantation d'organes; ${ }^{3}$ Departement de Néphrologie et Transplantation d'organes; ${ }^{4}$ Département de Néphrologie et transplantation d'organe; ${ }^{5}$ Département de Néphrologie et transplantation d'organertement de Néphrologie et transplantation d'organe, CHU Toulouse Rangueil, Toulouse, France

Background: ANCA-associated vasculitis (AAVs), including granulomatosis with polyangiitis and microscopic polyangiitis, are small vessel vasculitides. Modern treatments have greatly improved survival in AAV patients, but significant longterm morbidity and mortality such as cardiovascular disease (CVD) are still associated with this disease.

Objectives: The aim of our study was to assess the incidence, mortality and predictors of CVD in patients with AAVs

Methods: We conducted a retrospective study of AAV diagnosed in Toulouse France teaching hospital between 1981 and 2015. Patients with granulomatosis with polyangiitis (GPA) and microscopic polyangiitis (MPA) were included and classified, after thorough medical chart review, according to the criteria proposed by the Chapel Hill Consensus Conference. Eosinophilic granulomatosis with polyangiitis were excuded. A survival analysis was performed. Hazard ratios and the comparative morbidity and mortality figure (CMF) was calculated as the ratio of expected number of deaths in the standard population to those observed as well as predictors of CVD in patients with AAV were also assessed.

Results: We identified 361 subjects, only 125 met the inclusion criteria, 99 GPA and $26 \mathrm{MPA}, 65(42 \%)$ were men and mean age was $61.3 \pm 15.7$ years at CVD occurrence with a mean follow-up of $88.4 \pm 78.3$ months since diagnosis. Coronary artery disease (CAD) developed in 10 patients, and ischemic stroke developed in 9 patients (incidence rates of 8.5 per 1,000 person-years and 10.2 per 1,000 person-years, respectively). CAD incidence for AAV patients is two times more than in the general population, independent of age differences between the two populations using the Midi-Pyrénées county CAD registry as a reference (CMF of $1.96 ; 95 \mathrm{Cl} 0.88$ to 4.36 ). Ischemic stroke incidence for AAV patients is three times more than in the general population, independent of age differences between the two populations (CMF of 3.36 ; $95 \mathrm{Cl} 1.75$ to 6.46 ).

Smoking habits and history of CAD at AAV diagnosis was strongly associated with CAD occurrence (adjusted $\mathrm{HR} 8.8 ; 95 \mathrm{Cl} 2.12$ to $36.56, \mathrm{p}=0.003$ and adjusted HR 10.3; $95 \mathrm{Cl} 1.02$ to $104.5, \mathrm{p}=0.003$, respectively). An ENT flare (adjusted HR 0.12 ) was a independent protective factor for $C A D$ occurrence. We did not find any statistically significant associated factor with ischemic stroke occurrence in our cohort.

Using direct standardisation, the age adjusted mortality rate for the AAV cohort was 22.5 per 1,000 person-years and for the general population 10.2 per 1,000 person-years. This indicates that mortality for AAV patients is one and a half times more than in the general population, independent of age differences between the two populations (CMF of $1.56 ; 95 \mathrm{Cl} 1.02$ to 2.39 ).

Conclusions: Patients with AAV have a significantly increased risk of mortality and ischemic stroke and a non-statistically significant trend toward an increased risk of CAD. Monitoring for this complication and vigilance in modifying risk factors are particularly warranted in this patient population.

Disclosure of Interest: None declared

DOI: 10.1136/annrheumdis-2017-eular.6143
FRIDAY, 16 JUNE 2017

Cytokine taxonomy: reflection in the therapy of arthritides and other IMIDs

\section{OP0322 A NOVEL HIERARCHICAL RELATIONSHIP BETWEEN INTERLEUKIN-17A AND INTERFERON-ALPHA IS INDICATED BY ANALYSIS OF MULTIPLE CYTOKINES IN THE SERUM OF ADULT-ONSET STILL'S DISEASE AND BEHÇET'S DISEASE}

K. Sato, Y. Aizaki, Y. Araki, T. Mimura. Department of Rheumatology and Applied Immunology, Saitama Medical University, Saitama, Japan

Background: Adult-onset Still's disease (AOSD) and Behçet's disease (BD) are both systemic inflammatory diseases, the causes of which are largely unknown. They have been recently classified as autoinflammatory diseases, a group of diseases in which innate rather than acquired immunity plays important roles in their pathogenesis. As AOSD and BD are clinically distinct diseases, their cytokine networks should also be different.

Objectives: In this study, we attempted to quantify the levels of multiple cytokines in the serum of patients by utilizing a beads-array technique and ELISA, and then compared the serum cytokine profiles of the two diseases by factor analysis. We then sought to clarify the hierarchical relationship between interleukin (IL-) 17A and interferon (IFN-) a using peripheral blood mononuclear cells (PBMCs).

Methods: We quantified the serum levels of 10 cytokines (IFN- $\alpha$, IFN- $\gamma$, IL- $1 \beta$, IL-2, IL-4, IL-6, IL-10, IL-12p70, IL-17A and tumor necrosis factor $\alpha$ ) in 16 AOSD patients and 28 BD patients using multiplex bead array assays and $\mathrm{IL}-18$ along with ELISA. The data were then subjected to factor analysis. We next stimulated PBMCs from three healthy volunteers in vitro with class A CpG oligodeoxynucleotides (ODNs) in the presence or absence of IL-17A for 15 hours. We performed flowcytometric analysis to examine the expression of intracellular IFN- $\alpha$ in plasmacytoid dendritic cells (pDCs).

Results: Two factors were extracted from the factor analysis using the data on 8 cytokines that were detectable in the serum of the patients. IL-17A and IFN- $\alpha$, the levels of which showed a strong positive correlation in the serum of BD patients (Fig. A), were the main components of Factor 1, while Factor 2 consisted of IL-6, IL-10 and IL-18 (Fig. B). Patients were also plotted on a plane determined by Factors 1 and 2 according to each patient's factor scores. Those who were high in Factor 1 but low in Factor 2 were likely to be BD patients and vice versa, many of those who were high in Factor 2 but low in Factor 1 were AOSD patients. In terms of flowcytometric analysis, IL-17A alone did not induce IFN- $\alpha$ expression in pDCs, but it did substantially increase IFN- $\alpha$-positive pDCs induced by CpG ODNs.
(A)

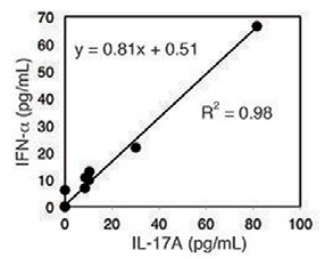

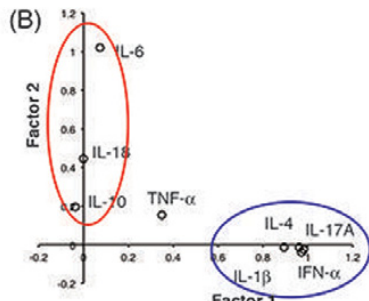

Conclusions: The cytokines examined were clearly separated into distinct groups by the factor analysis. Similarly, the AOSD and BD patients could be separated, although roughly. High levels of serum IL-6, -10 and -18 suggest AOSD while high levels of IFN- $\alpha$ and IL-17A indicate BD. To establish patterns of correlation among cytokines, it is important to focus on the cytokine concentrations in each patient, rather than the average cytokine concentrations in each of the diseases. In terms of the hierarchical relationship between IFN- $\alpha$ and IL-17A, a previous report suggested that IFN- $\alpha$ blocks IL-17A production in PBMCs from BD patients. Thus, we examined the effect of IL-17A on IFN- $\alpha$ production. It should be noted that the real stimulus for IFN- $\alpha$ release in BD is unknown. In addition, cells other than pDCs may be involved in the production of IFN- $\alpha$. Nevertheless, understanding the hierarchical relationship among cytokines should prove to be helpful in clarifying the pathogenesis of various inflammatory diseases.

Disclosure of Interest: None declared

DOI: 10.1136/annrheumdis-2017-eular.1678

\section{FRIDAY, 16 JUNE 2017}

\section{Personalised care for back pain}

\section{OP0323 DISCOPATHY ASSOCIATED WITH MODIC CHANGES IS NOT RELATED TO ANY INFECTIOUS PROCESS: A PROSPECTIVE MONOCENTRIC STUDY}

S. Ahmed Yahia ${ }^{1}$, J.-W. Decousser ${ }^{2}$, C.H. Flouzat-Lachaniette ${ }^{3}$, F. Robineau ${ }^{3}$, E. Audureau ${ }^{4}$, A. Hourdille ${ }^{1}$, F. Eymard ${ }^{1}$, X.-J. Chevalier ${ }^{1} .{ }^{1}$ Rheumatology; ${ }^{2}$ Microbiology; ${ }^{3}$ Orthopaedic; ${ }^{4}$ Public health, Henri Mondor hospital, Creteil, France

Background: Low back pain (LBP) is strongly associated with Modic changes. The 